\title{
A Qualitative Study Using Semi-Structured Interviews: Comparing the Views of Palliative Care Unit Patients and Clinicians on Corneal Donation Discussions
}

Chirag Patel ( $\square$ chiragpatel@yourclinicaljourney.com.au )

University of Adelaide

Gregory B Crawford

University of Adelaide

\section{Research Article}

Keywords: Palliative, medicine, cornea, qualitative, donation, interviews

Posted Date: January 3rd, 2022

DOI: https://doi.org/10.21203/rs.3.rs-1172675/v1

License: (a) (1) This work is licensed under a Creative Commons Attribution 4.0 International License.

Read Full License 


\section{Abstract}

Background: Blindness from corneal opacity accounts for $12 \%$ of cases of blindness worldwide. There is a severe shortage of corneas for donation worldwide for transplantation and research purposes. One group of individuals who could potentially be donors are those who die within the inpatient palliative care unit. The aims of the study were to 1 . determine the frequency of corneal donation discussion; 2 . determine whether inpatient palliative care unit patients and clinicians were aware of the potential for corneal donation discussions and 3. explore the attitudes and beliefs of inpatient palliative care unit patients and clinicians about corneal donation.

Methods: An exploratory qualitative study was designed where inpatient palliative care unit patients and clinicians were invited to a semi-structured interview. A total of 46 face to face interviews were undertaken involving inpatient palliative care unit patients (20) and clinicians (26) in three major inpatient palliative care units in South Australia.

Results: Very few patient participants were asked about corneal donations during their time in palliative care. Most inpatient palliative care unit clinicians did not bring up the topic as they felt other areas of care took precedence. Inpatient palliative care unit patients thought if inpatient palliative care unit clinicians did not raise the topic, then it was not important.

Conclusions: Findings suggest that patients are receptive to discussing corneal donations, but few discussions are occurring. There were some differences between patient and clinician views, such as preference about who raises the possibility of donation and when the discussion might occur.

\section{Key Statement}

\section{What is already known about the topic?}

Palliative care professionals do not raise the topic of corneal donation.

Palliative care patients would welcome the opportunity to discuss corneal donation.

\section{What are the new findings?}

This is the first Australian study to interview palliative care patients and clinicians and found that some views on corneal donation discussions did not align.

Patients would prefer to discuss this with doctors at a time when they are well enough but once they have accepted their illness.

Patients and clinicians were not adequately educated about corneal donations.

\section{What is their significance?}


Palliative care clinicians' and patients' views need to be aligned to positively impact the corneal donation program.

Corneal donation education programs for clinicians are imperative if they are to be comfortable in initiating corneal donation discussions.

Opportunistic discussion by any healthcare professional that has a rapport with the patient can potentially raise this topic with patients when they are well.

\section{Introduction}

Blindness from corneal opacity is a significant public health burden estimated to effect 4.9 million persons globally and accounts for about $12 \%$ of cases of blindness worldwide. ${ }^{1}$ There is a severe worldwide shortage of donated corneas for transplantation and research purposes. ${ }^{2}$ Although malignancy of solid organs is a contraindication for most organ donations, corneas are one of few tissues that patients with cancer can donate. Studies show that while intensive care patients have a high uptake of corneal donations, other patient groups show limited uptake. 3,4 It has been shown that palliative care units are a potentially unrealised setting to discuss corneal donation, and there is a general lack of awareness about corneal donation, and significant barriers to raising this topic. ${ }^{5}$ Although many studies have considered solid organ donation, there is still limited information on public perception of corneal donation, especially in western countries. ${ }^{6}$

Data from the 2020 South Australian Eye Bank Service shows a potential of 710 donors but only 32 actual donations (4.5\%). Since corneal grafting has to be undertaken within 12 hours, planning is necessary if a donation is to occur. ${ }^{7}$ Factors that impact rates of corneal donation include reduced awareness from patients and clinicians, skewed perceptions, and cultural issues. ${ }^{6,8}$

The relative infrequency of corneal donation conversations is already known in other countries such as the UK and USA. ${ }^{9}$ It has already been demonstrated that clinicians tend to have the same professional view as their personal view. ${ }^{10}$ There is a recent single-site study of patients' views on corneal donations, 11 a study that only involved a small number of patients, emphasising their views and timing about how the topic might be discussed. What is not known in comparing the views of clinicians and patients about corneal donation specifically around information is: when to ask, how to ask and what patients would like to know? We aim to compare the views of clinicians and patients from the three major palliative care units in South Australia about what are believed to be the essential areas in corneal donation discussions and determine what the most important issues are? Patient attitudes to corneal donation have been reported in other countries, but this is, to our knowledge, the first in Australian palliative care patients. 9,12

\section{Methods}

The aims of the study were to 
1. Determine the frequency of corneal donation discussions

2. Determine whether Palliative Care Unit (PCU) patients (PCUP) and clinicians (PCUC) (consisting of doctors, nurses, and social workers) were aware of the potential for corneal donation discussions

3. Explore the attitudes and beliefs of PCUP and PCUC about corneal donation.

This is an exploratory study using qualitative methods to establish common themes. The study was conducted in three specialist palliative care units in South Australia between January 2020 and September 2020. Participants recruited were inpatients, and clinicians, also referred to as patients and clinicians, consented to take part in a semi-structured interview, after which thematic analysis of transcripts was undertaken using grounded theory. Interviews were conducted either face-to-face or by telephone, depending on participant preference and restrictions imposed by the COVID-19 pandemic. With regards to telephone interviews, consent was taken over the phone and.an electronic consent form was sent to the participant and a signed copy was sent back to the researcher via email. There were some breaks in the recruitment of PCUP due to the COVID-19 pandemic.

A census sampling method was used to recruit the PCUP, whereby the nursing team leader was asked to provide an information leaflet to those patients admitted to the PCU and met the inclusion criteria, inviting them to participate. If patients were interested, the team leader notified the researcher that they had provided consent to be contacted. These patients were given more information, and an interview was arranged. If the team leader deemed the patient who had previously agreed was found to be cognitively impaired or too unwell, they were not approached. The PCUC were approached by email with an attached information sheet, and if they agreed, a time was organised for the interview to take place. The study continued until saturation of themes was achieved. A preliminary analysis was carried out on completed interviews to determine data on emerging themes during data collection.

The inclusion criteria were as follows:

- PCUC (doctors, nurses, and social workers)

- PCUP over the age of 18.

- English speaking

- Be able to give informed consent

- Be able to talk for up to 45 minutes

All semi-structured interviews were conducted one-to-one by a single author (CP) at the PCU (or over the phone for PCUC) and had no clinical role in any recruitment sites. At the time of the research conception, he worked as a palliative medicine registrar, but during data collection, he was working as a rural generalist. An interview guide was used as a prompt of key areas to explore (see Additional File 1.). This was based on a similar guide piloted and used by Wells et al. ${ }^{3}$ PCUP, and PCUC gave written consent for the interviews to be recorded digitally. If participants requested information about possibly becoming a corneal donor, they were provided with an information pamphlet from the South Australian Eye Bank 
Service, and the treating team was notified with the patient's consent about their interest. PCUP and PCUC were aware they had the right to withdraw at any time.

The interview recordings were transcribed verbatim by the lead author and stored on a passwordprotected computer. Any identifying information was removed. Data analysis was conducted using thematic analysis and grounded theory. ${ }^{13}$ In addition, words, context, consistency, frequency, intensity and specificity of comments were analysed. Qualitative data analysis software was not used; however, Microsoft applications Word and Excel were used to sort data and search and analyse themes.

Human Research Ethics Committee granted ethics at Central Adelaide Local Health Network. (Approval Number 12054)

\section{Results}

A total of 25 patients were approached, of which 20 patients consented to be interviewed. Invitations were sent to the whole department at the three PCUs, and from these, 26 clinicians consented and were interviewed. Five clinicians were interviewed over the telephone; details of which PCUC were interviewed and where can be found in Table 1. Interview times ranged from 16-35 minutes duration with a mean time of 22 minutes. The most frequent life-limiting illness was breast cancer. Participant demographic data can be seen in Table 2 .

Table 1

Breakdown of patient and clinician numbers across the three palliative care units

\begin{tabular}{|c|c|c|c|c|}
\hline & \multirow[t]{2}{*}{ Patient, n (\%) } & \multicolumn{3}{|c|}{ Clinician, n (\%) } \\
\hline & & Doctor & Nurse & Social Worker \\
\hline PCU 1 & $9(45 \%)$ & $3(30 \%)$ & $3(27 \%)$ & $1(20 \%)$ \\
\hline PCU 2 & 7 (35\%) & $4(40 \%)$ & $5(45 \%)$ & $2(40 \%)$ \\
\hline PCU 3 & $4(20 \%)$ & $3(30 \%)$ & $3(27 \%)$ & $2(40 \%)$ \\
\hline
\end{tabular}


Table 2

Patient and clinician demographics

\begin{tabular}{|ll|}
\hline Clinician & \\
\hline Gender (\%) & $10(38.4 \%)$ \\
Male & $16(61.6 \%)$ \\
Female & \\
\hline Mean age (years) & 41.3 \\
\hline Mean palliative care experience (years) & 6.4 \\
Patient & \\
Gender (\%) & $9(45 \%)$ \\
Male & $11(55 \%)$ \\
Female & \\
Mean age (years) & 68.2 \\
Life limiting illness, $n$ (\%) & $3(15 \%)$ \\
Breast Cancer & $2(10 \%)$ \\
Pancreatic Cancer & $1(5 \%)$ \\
Bladder Cancer & $1(5 \%)$ \\
Endometrial Cancer & $1(5 \%)$ \\
Lung Cancer & $2(10 \%)$ \\
Metastatic Squamous Cell Cancer & $2(10 \%)$ \\
Prostate Cancer & $1(5 \%)$ \\
Leukaemia & $1(5 \%)$ \\
Myeloproliferative Disorder & $2(10 \%)$ \\
Cervical Cancer & $1(5 \%)$ \\
Renal Cell Cancer & $1(5 \%)$ \\
End-Stage CoPD & $1(5 \%)$ \\
\hline
\end{tabular}


Themes that emerged from patient interviews are referenced in Table 3. Almost all the patients interviewed stated they would like to discuss corneal donation (94\%) and donate their corneas (92\%). A large majority of PCUPs (94\%) thought the discussion would benefit them, but only $76 \%$ of clinicians thought it beneficial. Only $11 \%$ of patients were asked about corneal donations while being under the care of the palliative care team. 
Table 3

Patient themes

\section{Themes Quotes}

Knowing "I don't know the full details of corneal donation - I know that someone will come and and awareness get the parts from me when I die" (Patient 1a)

"I don't know anything" (Patient 3a)

"I don't want to know more because I won't even be here anyway" (Patient 7b)

"I think my cancer would affect me donating, but I wouldn't have a complete clue on that one. I think logically, if there is cancer in the body, then it can't really be used by anyone" (Patient 4c)

"I would have thought that people with cancer can't donate any parts of their body. I thought that if so many parts of their body are diseased when they've got the spread of cancer, I just assumed there wouldn't be anything that was useful" (Patient 6a)

Altruism "If I can help someone such as those involved in research or any people to see again, then it will be worthwhile" (Patient 3a)

"Any part of the body that could be used to help someone else because it is not damaged in any way, I think is beneficial for everyone in general. This could be important for myself, my family and the recipient" (Patient 4b)

"Personally, helping others would be the main reason - the fact that I can potentially give sight to someone else is fulfilling for me, even though I know I won't be around to see it afterwards" (Patient 6b)

Choosing "I do feel it is important, some people may get distressed by it, but others may surprise you" (Patient 5a)

"If we are not asked, then we will never know" (Patient 5a)

"My families' views wouldn't change my view on donating my corneas" (Patient 6a)

"I had an experience which I regret really - I declined donating corneas when my sisterin-law died. Since then, I have really thought about how it could have helped others" (Patient 9a) 


\section{Themes Quotes}

Informing "I feel discussing corneal donations important early on is not that important, but it is important to bring up at some point, maybe after building some kind of rapport with me" (Patient 2c)

"I believe that once you have been diagnosed and you know the reason that everyone is helping you to make your life comfortable for as long as possible, I think in those moments is a good time to discuss those points" (Patient 6a)

"Given the fact, there have been no conversations with me; a trigger would be needed so maybe when I had my ACD discussion that would have been an ideal opportunity or even when I am discussing goals of care when I come into hospital" (Patient 7b)

I would like to have discussions at home about something so sensitive" (Patient 4a)

"The palliative care unit is a very calm and peaceful environment, and so it would be naturally a good choice in terms of location to bring up a topic like this" (Patient 2a)

I think the social worker would be the best person to discuss this with me, as they know me well and know all my wishes, but so do the doctors and nurses" (Patient 5b)

"I would like a palliative care doctor to discuss this with me - I would say that I have a good rapport with my palliative care doctor, so that would fit in well" (Patient 1a)

"I am old school; if you want to ask someone something, then the only way to ask is to look them in the eyes and ask them" (Patient 2c)

"I would definitely like some written information on this, but the conversation through direct communication with another person is far more meaningful" (Patient $5 b$

Skills required to discuss
"I do think that they have to have good communication skills and be empathetic, but also they have to know what is involved" (Patient 7a)

"They should have a good broad knowledge of the area and good communication skills" (Patient 9a)

"They should have a sound knowledge of the subject so they can answer any questions; they have to communicate effectively, be empathetic and listen to what I have to say rather than push their views" (Patient $3 b$ )

"I have never been asked about corneal donation in my time with palliative care, even on admission here" (Patient 2d).

"I don't think it is something that we think of immediately in the chaos of what is going on" (Patient 1e).

Twenty per cent of clinicians interviewed expressed that they discuss corneal donation with their patients; however social workers ask more often than doctors and nurses. A higher proportion of clinicians want to ask about corneal donation (76.9\%) but do not. Regarding knowledge, both patients and clinicians had limited knowledge of corneal donation, with only $20 \%$ of patients and $34.6 \%$ of clinicians having some form of knowledge on this subject. (Table 4) 
Table 4

Comparison of patients' and clinicians' views on corneal donation

\begin{tabular}{|c|c|c|c|c|c|c|c|c|}
\hline & \multicolumn{2}{|c|}{ Patient } & \multicolumn{2}{|c|}{ Doctor } & \multicolumn{2}{|c|}{ Nurse } & \multicolumn{2}{|c|}{$\begin{array}{l}\text { Social } \\
\text { worker }\end{array}$} \\
\hline & Yes & No & Yes & No & Yes & No & Yes & No \\
\hline Do they know about the processes? & 4 & 16 & 3 & 7 & 4 & 7 & 2 & 3 \\
\hline Have discussions ever been initiated? & 2 & 18 & 1 & 9 & 2 & 9 & 2 & 3 \\
\hline Is it essential/beneficial to discuss? & 18 & 2 & 6 & 4 & 7 & 4 & 5 & 0 \\
\hline $\begin{array}{l}\text { Would clinicians like to ask, or patients like to be } \\
\text { asked? }\end{array}$ & 19 & 1 & 8 & 2 & 7 & 4 & 5 & 0 \\
\hline
\end{tabular}

Knowing and awareness

Many PCUPs reported that they did not have knowledge around the process of corneal donation (80\%). Most knowledge was framed in terms of altruistic assistance to others. While most PCUPs wanted to know more about corneal donation, one did not want to know more as they "would not be alive". Many PCUP $(60 \%)$ thought their cancer diagnosis would mean they could not donate or were not entirely sure if they could.

Altruism

All the patients expressed their desire to "help" others through donation.

Choosing

All patient participants said they would like to discuss corneal donation, but they had not had the opportunity yet. Whilst some participants recognised that they could raise the subject, they did not know enough about it and felt it was possibly not crucial because the clinician did not. They also felt that their families should be involved. Most of the participants revealed that while it was important for them to be involved, their families' decisions would not affect their final decision.

Informing - When, where, who and how?

There was a mixed response about when the discussion should occur, but the overwhelming majority stated it would be best once the patient had accepted the diagnosis. There was recognition that while it is a sensitive subject, rapport with the patient may alleviate this aspect. As long as no distressing symptoms were present, anytime would be reasonable. Interestingly two patients mentioned the advance care directive discussion as a trigger for this topic. Most patients would like to discuss it while an inpatient, but of the ones who suggested an alternative place, there was an approximately equal spread about those options. While $30 \%$ of patients felt the doctor would be the best equipped to initiate discussions, $60 \%$ felt other clinicians or someone they have a rapport with might be a better option. Ten 
per cent believed that it did not matter who had the conversation with them. However, $50 \%$ of participants felt that written, video and face to face information available should be used to initiate discussion about corneal donation, while $35 \%$ felt that face to face contact was preferred. (Tables $5,6,7.8$ )

Table 5

Patient and clinician views on the timing of the discussion

\begin{tabular}{|lllll|}
\hline When is the best time to discuss this? & Patient & Doctor & Nurse & Social worker \\
\hline Early in illness & 2 & 3 & 4 & 1 \\
\hline After the patient has accepted their illness & 13 & 2 & 3 & 3 \\
\hline When having discussions around goals of care & 2 & 4 & 1 & 1 \\
\hline Towards the end of life & 3 & 0 & 2 & 0 \\
\hline Does not matter & 0 & 1 & 0 & 0 \\
\hline Never & 0 & 0 & 1 & 0 \\
\hline
\end{tabular}

Table 6

Patient and clinician views on preferred site of discussion

\begin{tabular}{|lllll|}
\hline The preferred site of discussion & Patient & Doctor & Nurse & Social worker \\
\hline Palliative care unit & 8 & 3 & 4 & 1 \\
\hline Outpatient department & 2 & 2 & 3 & 1 \\
\hline Home & 3 & 0 & 3 & 3 \\
\hline Other & 2 & 0 & 0 & 0 \\
\hline Does not matter & 4 & 5 & 1 & 0 \\
\hline
\end{tabular}

Table 7

Patient and clinician views on a preferred person to initiate discussion

\begin{tabular}{|lllll|}
\hline Is there a preference for who initiates the discussion? & Patient & Doctor & Nurse & Social worker \\
\hline Doctor & 6 & 1 & 4 & 2 \\
\hline Nurse & 5 & 3 & 2 & 2 \\
\hline Social worker & 3 & 3 & 2 & 0 \\
General Practitioner (GP) & 1 & & & \\
With whom there is best rapport & 3 & 1 & 3 & 1 \\
\hline Does not matter & 2 & 2 & 0 & 0 \\
\hline
\end{tabular}


Table 8

Patient and clinician views on how to initiate discussion

\begin{tabular}{|lllll|}
\hline Preferred mode of discussion & Patient & Doctor & Nurse & Social worker \\
\hline Face to face discussions & 7 & 4 & 6 & 3 \\
\hline Telephone & 0 & 0 & 0 & 0 \\
\hline Written literature & 3 & 0 & 3 & 0 \\
\hline All three & 10 & 5 & 2 & 2 \\
\hline Does not matter & 0 & 1 & 0 & 0 \\
\hline
\end{tabular}

\section{Skills required to discuss}

Two common words that arose frequently in conversation was "communication" and "empathy". There was also frequent mention that it is important that those who discuss corneal donation should have adequate knowledge about the subject.

\section{Clinician Themes}

Clinician themes that emerged are referenced in Table 9. Most of the clinicians appreciated how corneal donations could benefit others in terms of sight-saving and the donor by giving them a sense of purpose and a meaning to their death. The concept of "legacy" was raised frequently in discussions. 


\section{Themes Quotes}

Gatekeeping "As long as they have an understanding about the benefits and reasons, then I think it would be great for the patient and people out there - there are a lot of people out there that would value donating their corneas for transplant or research" (Social worker 1a)

"I think the way we approach our palliative care patients is to give them control and choices, and this would be another aspect of this. So, I think this is actually really appropriate" (Social worker 1b)

"From the science point of view, it is a good opportunity to find out more on corneas via research, but from the patient point of view, there will be some patients who have too much going on and have so much distress in their journey and then having this discussion will put too much burden on them" (Nurse 3c)

"We have lots of patients who are newly diagnosed and asking about this can put much pressure on them and cause distress. We could end up upsetting them more" (Nurse 2b)

"Not really because I thought we were oversupplied with corneas. A few years ago, I notified the eye bank about a dying patient in ICU, and they said we have enough corneas and so said not to worry. Since then, I have not bothered with regards to this" (Doctor 3c)

"I must admit I haven't thought about what I feel" "I would want to know about the literature of what the patient perception is around this subject before I discuss something like this" (Doctor 2b)

Knowing and awareness
"I am not familiar with the process of corneal donation but would like to know about it more" (Doctor 3a)

"Building more awareness about this topic is important especially when a patient is cognitively aware and able to make decisions" "For me personally we have never had any education about it and maybe medical school is a good place to start" (Doctor 3b) 


\section{Themes Quotes}

Informing "We should do it on admission or even in the community - give them enough time to think about it before the terminal phase. Early on in the illness is the best time to discuss this" (Nurse 2a)

"I feel it is best to discuss this when we have built a rapport with the patient - when we know more information about the patient" (Doctor 2a)

"Depends on the mindset of the patient - if they are accepting of their palliative condition. If someone can't even accept dying, then that is not a good time" (Social worker 2b)

"I probably sound a little old fashioned, but there must be a sense of what we know about the patient and their illness first; I would have thought it would be when the patient is in the incurable phase and probably at that advanced care planning stage" (Doctor 3c)

"At least not when they are first diagnosed or when they are terminal phase. Probably when their symptoms are more under control, and they are past their denial stage" (Nurse 4a)

"I think if you asked me 12 months ago, then often these conversations would be best face to face, but the last six months, I have used video telehealth a lot, and I would be comfortable to bring this up through this medium" (Doctor 3a)

Skills required to discuss

Barriers to discussing
"Communication is a big thing here, I believe, as well as empathy" (Nurse 3c)

"They need to be knowledgeable and confident in sensitive discussions, so communication and being empathetic springs to my mind" (Doctor 2c)

"No concerns of raising this topic if it is well explained. However, some staff can be reluctant, so they need education - maybe they are scared to bring it up. The other concern is if it is dropped suddenly onto a patient without an explanation" (Nurse 4a)

"I don't bring it up myself, but for a long time, I would like the question to be put on an admission proforma. It's a sensitive issue, and often there are a number of things that are happening on admission to a palliative care unit, and so this takes a back seat" (Nurse 2b)

"I don't bring up discussions myself - at the moment; it's just not part of the culture or the admission process" (Nurse 3c)

"I don't do it all the time as we have so many other things to discuss, and to be honest, in the centres we are working in at the moment, it is not embedded in our everyday practice" (Doctor 2c)

"If I am educated and exposed to the topic, then I don't think I would have a problem; the key thing is education" (Social Worker 2b)

"There is a benefit to the visual integrity of the person receiving the cornea. "There are also benefits to the patient - may give them a sense of purpose - other parts of their bodies may be deteriorating, but there are still parts that are of significant value to others" (Doctor 2a)

"I think a sense that the patient's valuable organ could be useful and despite knowing that their life is limited, they can assist others to live better with improved vision" (Nurse 1a) 
"We certainly know that a lot of people want to contribute, and patients will say if someone can learn something from my corneas, that will make them feel better" (Doctor $3 b$ )

Gatekeeping

During interviews, some clinicians felt that it could be detrimental to discuss something so sensitive with patients and, for that reason, may not be beneficial to them. One doctor wanted to research the available literature of patient perception on this subject before they would broach it. Overall, clinicians felt it was in the best interests of the patient to have discussions. One doctor mentioned that he had actively brought this topic up previously but was told by the eye bank that they have enough corneas, so not to worry about this. This was a deterrent for him to bring up the topic.

Knowing and Awareness

Interestingly only a handful of clinicians were confident enough to discuss corneal donations with their patients (30\%). One nurse was advocating for workshops and education around this topic within their department. (Table 4.).

Informing - when, where, how and who?

Doctors and nurses had divided opinions on when to raise the topic of corneal donation, with no clear majority option. However, $60 \%$ of social workers felt it was best to raise this after the patient had "accepted their illness". However, there was a consensus that leaving it too late was not ideal either. One nurse and one social worker commented on initiating discussions during advance care directive preparation.

Half of the doctors interviewed believed that it did not matter where the discussion about corneal donation occurred. In comparison, nurses were divided about whether the best location was the palliative care unit, outpatient department or home. Social workers felt that home was the best place to discuss this issue. The majority of clinicians felt that face to face discussions was the best option; however, $50 \%$ of the doctors felt that a combination of written, video and face to face discussions would be ideal. Interestingly, most clinicians stated that their colleagues would be the best person to have the discussion, i.e., doctors felt that the nurses and social workers would be ideal; nurses felt doctors and social workers would be ideal. (Tables 5,6,7,8.)

Skills required to discuss

All clinicians felt that good communication such as active listening, non-verbal communication and clarity was vital. One third raised empathy and knowledge as additional skills required to discuss this topic.

Support required 
All clinicians thought that with patient permission, family members, substitute decision-makers or next of kin being present at the conversation was a good idea.

Barriers to discussing

Clinicians considered that barriers to discussing corneal donation may be that patients were dealing with other more pressing issues, making this issue not relevant or not a priority. In addition, two-thirds of clinicians specified their lack of knowledge on the topic, thus creating a further barrier to discussion.

\section{Discussion}

Previous research has shown differing and conflicting results about whether palliative care patients would donate their corneas. ${ }^{11,12}$ This study provides further evidence that palliative care patients depending on their illness trajectory, are open to having these kinds of discussions and potentially donating their corneas. This study further supports evidence that even raising the topic of donation is unlikely to be instigated for palliative care patients. 3,12,14 Even though clinicians may want to raise this topic, the barrier of time, fear of removing hope, or even altruistic beliefs that are gatekeeping obstructions may occur. ${ }^{14}$ And some patients believe that if the clinician does not raise such a topic, then they believed it was not important to the clinician or the patient, meaning a total disconnection between patient and clinician expectations.

There are studies reporting that patients would like to discuss donations at the time of PCU admission, ${ }^{15,16}$ but not during the first admission. ${ }^{17}$ This study revealed that patients feel comfortable in the PCUP environment, and the majority deemed this an appropriate place for discussion. Doctors and nurses also concurred, but social workers felt the patient home was perhaps more suitable. Social workers are frequently involved in patient care in their homes, being more aware of home as a preferable option.

Patients generally felt that a doctor or nurse should initiate the conversations, with the majority indicating doctors. Previous studies have shown that having a rapport is essential for the patient. ${ }^{12}$ And whilst three patients in this study felt this way, there was a consensus that patients who chose a doctor or a nurse indicated that they had a rapport with the chosen IPUC. Walker et al. believed that doctors were not ideal given the length of their relationships with patients are shorter. ${ }^{12}$ Interestingly, most doctors felt they were not best placed to discuss this topic, which was different from the patient's viewpoint. This reiterates the importance patients place on doctors to initiate and guide them through complex topics.

The method of delivery is vital to ensure a positive outcome for patients. Many patients reported face to face as an ideal medium to discuss and converse, and the clinicians also felt this way. However, most patients felt that a combination of face to face, telephone discussion, and written literature was preferred. Walker et al. and Cochran et al. reported face to face discussion was preferred over other methods. ${ }^{11,15}$ 
While anyone can discuss this topic in theory, patients felt that specific criteria had to be met by the person who would initiate the discussion. Notably and obviously, the ability to communicate sensitively and empathetically was essential. Clinicians also felt that the ability to communicate and deliver the discussion well was the key. It cannot be overstated that good communication can reduce the refusal of donation. ${ }^{18}$ On top of this, a common opinion was that they had to be adequately knowledgeable on the subject. Interestingly for clinicians, this was a common barrier to discussion. Many clinicians and indeed patients knew very little about the whole process, as elicited in other studies. ${ }^{11,12,14,15}$

This study demonstrates that many patients and clinicians are ignorant or have real misconceptions about corneal donation. It also displays the value that patients impart to clinicians in asking about and understanding organ donation and in particular corneal donation. Patients had a preference that a doctor would be the best person to raise the topic, yet doctors thought otherwise. This emphasises that clinicians need to understand the patient's preference to improve the patient's quality of care. ${ }^{19}$ Whilst clinicians knew that a cancer diagnosis was not a contraindication for corneal donation, many patients did not understand that they could donate their cornea despite a diagnosis of cancer. This assumption has been supported in previous studies on donation in palliative care. ${ }^{15,17}$

Commonly, the theme of altruism or "doing good" was expressed by patients, and the positive effect donation could have on themselves was evident in discussions. However, not many patients discussed the benefit for research. Altruistic behaviour is not a new phenomenon, and evidence shows that helping others positively affects mental health. ${ }^{20}$ Mental health in palliative care patients is an important area of provision of care. Providing them with an opportunity to help others or framing it this way could be an avenue to pursue. Clinicians also held the same view and felt it provided patients an opportunity of a legacy and possibly being seen as empowering. Studies from other countries have found similar themes. ${ }^{17,21}$ A large study in the UK considering clinicians' views was in line with this study findings, but there were barriers to discussing this topic. ${ }^{14}$

Choosing whether to donate or not is a challenging and very personal conversation. Most of the patients described that they would want support from family and friends for such discussions. However, there were varying thoughts about whether their family and friends' views would affect their own. This is supported by other recent research. ${ }^{17}$ One study has shown that families generally are receptive to corneal donation discussions and find the concept to be positive. ${ }^{22}$ Clinicians' views also aligned with those of the patients regarding support being present.

Clinicians are known at times to be gatekeepers in care and even in palliative care. ${ }^{14}$ In this study, nurses, raised concerns about such discussions being detrimental to the patient, despite evidence to the contrary. However, certain factors may dampen this impact, such as the timing of the conversation and the communication methods used. ${ }^{23}$ The timing of such a discussion may play a major role in whether the patient opts to donate or not. Naturally, the patient must be receptive to the subject being discussed and given that it is a topic of sensitivity, some level of rapport has to be present. This was quite apparent in 
many patient views. Not many patients chose to have this discussion towards the end of life. Equally, not many patients chose to have this discussion early. This mirrored other studies. ${ }^{11}$ In a 2015 survey about general organ donation, it was noted that over $50 \%$ of patients who expressed a preference would prefer to be asked their views on donation during their first outpatient appointment, ${ }^{12}$ however, this may not be representative of contemporary views. The majority of the social workers agreed with patients, but nurses and doctors were divided in about when an appropriate time was to raise this topic. Literature suggests that healthcare professionals believe that patients should be cognitively and physically well at the time of discussion. ${ }^{23}$

This study has several limitations. The patient population was generally old and may not represent the views of a younger patient cohort, who may potentially have a greater level of distress. Because patients were admitted in a palliative care unit, this may have influenced their decisions about where the discussion should occur, i.e., creating a location bias. Patients who did not speak proficient English were excluded, and the possible cultural elements were eliminated, creating a potential selection bias.

\section{IMPLICATIONS OF THE RESEARCH FOR CLINICAL PRACTICE}

This study demonstrates that corneal donation discussions are not distressing for patients. Palliative care doctors are ideally placed to discuss this topic, but adequate education is imperative to give them the confidence and knowledge to initiate discussions. ${ }^{24,25}$ Advance care planning provides an opportunity for this discussion, or a trigger reminder on an admission proforma could be an alternative option. $\mathrm{A}$ participant mentioned that education for patients could be presented at clubs and a situation where nonhealthcare professionals could be utilised for discussions. Non-palliative care health professionals may also be ideal if a rapport with the patient exists. Further research is required into looking at minority groups that historically have had lower donation rates. ${ }^{26}$

\section{Conclusion}

It was evident from this study that there were some incongruities between patient and clinician views concerning the discussion of corneal donations. To achieve a satisfactory outcome in patient care, generally, the patient perspective and clinician perspective should be aligned. This study would suggest that the option of corneal donation is not being raised with patients as frequently as patients might desire. This study identifies barriers such as lack of education and not even considering the topic when other aspects of care might take precedence. There is a need for more education for clinicians. Education is an essential aspect of any donation program, but with corneal donations, it becomes more imperative given that 1) patients expect this and 2) malignancy is not always a contraindication. It has been shown that clinician education can lead to an increase in the discussion, which is another reason for initiating education. Knowing that patients, in general, want to engage in discussions around corneal donation, 
there are numerous opportunities to discuss the option throughout the patient care path. However, choosing the right time is paramount.

In summary, patients prefer to have this conversation face to face with a clinician who has a rapport with them in a palliative care unit at a time where the illness has been "accepted". A reminder trigger on an admission form for the nurses and doctors may be worth pursuing as an avenue, thus aiding as a reminder.

\section{Declarations}

\section{Author Contributions}

Chirag Patel was involved in the concept, design, implementation, data collection and subsequent manuscript preparation.

Gregory B Crawford was involved in the concept, design, and subsequent manuscript preparation.

\section{Consent for publication}

Consent was sought from all participants to publish findings of this study.

\section{Funding}

There was no funding required for this project.

\section{Competing Interests}

The authors declare there are no competing interests.

\section{Ethics approval and consent to participate}

Human Research Ethics Committee granted ethics at Central Adelaide Local Health Network. (Approval Number 12054).

Data was collected in accordance with the tenets of the Declaration of Helsinki.

Consent was obtained from all participants included in this study, prior to commencement of data collection.

\section{Availability of data and materials}

The datasets generated and/or analysed during the current study are not publicly available due to consent not being granted from participants to distribute all the data due to personal reasons, but certain aspects of the data is available from the corresponding author on reasonable request 


\section{References}

1. Pascolini D, Mariotti SP. Global estimates of visual impairment: 2010. Br J Ophthalmo/ 2012; 96(5): 614-8.

2. Williams AM, Allingham RR, Beckwith HS et al. Patient and family attitudes about an eye donation registry for research. Curr Eye Res 2013; 38(9): 945-51.

3. Wells J, Sque M. 'Living choice': the commitment to tissue donation in palliative care. Int J Palliat Nurs 2002; 8(1): 22-7.

4. Miller $C$, Breakwell R. What factors influence a family's decision to agree to organ donation? A critical literature review. London J Prim Care (Abingdon) 2018; 10(4): 103-7.

5. Williams AM, Muir KW. Awareness and attitudes toward corneal donation: challenges and opportunities. Clin Ophthalmol 2018; 12: 1049-59.

6. Lawlor M, Kerridge I, Ankeny R et al. Specific unwillingness to donate eyes: the impact of disfigurement, knowledge and procurement on corneal donation. Am J Transplant 2010; 10(3): 65763.

7. Gillon S, Hurlow A, Rayment $C$ et al. Eligibility for corneal donation within the hospice population. Palliat Med 2010; 24(5): 551-2.

8. Ralph AF, Alyami A, Allen RDM, et al. Attitudes and beliefs about deceased organ donation in the Arabic-speaking community in Australia: a focus group study. BMJ Open 2016; 6(1): e010138.

9. Madi-Segwagwe BC BM, Myall M, and Long-Sutehall T. Barriers and Facilitators to Eye Donation in Hospice and Palliative Care Settings: A Scoping Review. Palliat Med Rep 2021; 2(1): 175-87.

10. Al-Qurainy R, Prentice W. Corneal donation in palliative care: a doctor's perspective. BMJ Support Palliat Care 2011; 1(2): 205-

11. Walker $\mathrm{L}$, Neoh $\mathrm{K}$, Gilkes $\mathrm{H}$ et al. A qualitative study using semi-structured interviews of palliative care patients' views on corneal donation and the timing of its discussion. Palliat Med 2018; 32(8): 142837.

12. Edwards P. Corneal donation within palliative care: a review of the literature. Int J Palliat Nurs 2005; 11(9): 481-6.

13. Braun V, Clarke V. Using thematic analysis in psychology. Qual Res Psychol 2006; 3(2): 77-101.

14. Gillon S, Hurlow A, Rayment $C$ et al. Obstacles to corneal donation amongst hospice inpatients: A questionnaire survey of multi-disciplinary team member's attitudes, knowledge, practice and experience. Palliat Med 2012; 26(7): 939-46.

15. Cochran L, Shorthose K. How and when to ask cancer patients with a palliative diagnosis about corneal donation. A questionnaire based study. BMJ Support Palliat Care 2021; 11(Suppl 1): A34-A.

16. Callander S. Tissue donation: an option after death. Palliative Care Today 1998; 7 (11): 4-5. 
17. Walker $\mathrm{L}$, Neoh $\mathrm{K}$, Gilkes $\mathrm{H}$ et al. A qualitative study using semi-structured interviews of palliative care patients' views on corneal donation and the timing of its discussion. Palliat Med 2018; 32(8): 142837.

18. Siminoff LA, Traino HM, Genderson MW. Communicating Effectively about Organ Donation: A Randomised Trial of a Behavioral Communication Intervention to Improve Discussions about Donation. Transplant Direct 2015; 1(2).

19. Say RE, Thomson R. The importance of patient preferences in treatment decisions--challenges for doctors. BMJ 2003; 327(7414): 542-5.

20. Schwartz C, Meisenhelder JB, Ma Y et al. Altruistic social interest behaviors are associated with better mental health. Psychosomatic Medicine 2003; 65(5).

21. Yew Y-W, Saw S-M, Pan JC-H, et al. knowledge and beliefs on corneal donation in Singapore adults. Br J Ophthalmol 2005; 89(7): 835-40.

22. Carey I, Forbes K. The experiences of donor families in the hospice. Palliat Med 2003; 17(3): 241-7.

23. Wale J, Arthur A, Faull C. An analysis of knowledge and attitudes of hospice staff towards organ and tissue donation. BMJ Support Palliat Care 2014; 4(1): 98-103.

24. Roach $\mathrm{R}$ and Broadbent A. Eye donation in Sydney metropolitan palliative care units. $J$ Palliat Med 2010; 13(2): 121-3.

25. Callender CO, Washington AW. Organ/tissue donation the problem! Education the solution: a review. J Natl Med Assoc 1997; 89(10): 689-93.

26. Callender CO, Miles PV. Minority Organ Donation: The power of an educated community. J Am Coll Surg 2010; 210(5): 708-15.

\section{Supplementary Files}

This is a list of supplementary files associated with this preprint. Click to download.

- AdditionalFile1.docx

- SupplementaryInformation.docx 